\title{
Un ejercicio de reestructuración curricular en la educación superior para aportar a la formación ciudadana en Colombia: un caso en el municipio de Zipaquirá*
}

\author{
Santiago Torres**
}

Fecha de recepción: 27 de abril de 2015

Fecha de revisión: 5 de mayo de 2015

Fecha de aprobación: 29 de mayo de 2015

\section{Resumen}

Este trabajo se basó en la reestructuración curricular del curso "Comunicación, ciudadanía y convivencia" del programa de Comunicación Social (periodismo) de la Universidad Minuto de Dios, del municipio de Zipaquirá, Colombia. Este contexto se caracteriza por ser rural, particularidad que captó mi atención como investigador por indagar sobre cuál era la manera más adecuada de diseñar el curso, analizar cómo los estudiantes asumen los conceptos allí trabajados y mirar si estos eran concretados en acciones reales después de haberlos aprendido en clase.

* Artículo de investigación científica y tecnológica. Este artículo muestra los resultados de mi tesis de maestría en Educación de la Universidad de los Andes titulada "Analisis curricular del curso Comunicación Ciudadanía y Convivencia perteneciente al Programa de Comunicación Social-Periodismo del Centro Regional de Educación Superior Zipaquirá de la Corporación Universitaria Minuto de Dios".

** Magister en educación de la Universidad de Los Andes, Comunicador social periodista de la Universidad Santo Tomás con énfasis en educación. Profesor universitario desde el año 2011 en la Universidad Minuto de Dios Sede Principal. Dirección de correspondencia: Calle 81b \# 72 B-70. Bogotá, Colombia. Programa Comunicación Social-Periodismo. Correo electrónico: storres@uniminuto.edu 
Este trabajo conllevó a un análisis conceptual de las competencias ciudadanas propuestas en clase y un abordaje crítico sobre si los estándares propuestos por el Ministerio de Educación Nacional son o no un buen insumo para la educación ciudadana en este contexto.

Como resultado de esta investigación se logró identificar la principal ruta curricular del curso basado en las características del contexto y las particularidades de los estudiantes. Asimismo permitió entender cómo ellos se apropiaron de las competencias ciudadanas desarrolladas en la clase y cómo estas se convirtieron en acciones reales en sus contextos.

\section{Palabras clave}

Ciudadanía, competencias, currículo, educación superior, aprendizaje.

\section{An exercise of curricular restructuring in higher education to contribute to citizenship education in Colombia: a case study of the municipality of Zipaquirá}

\section{Abstract}

This work is based on the course curriculum restructuring "Communication, citizenship and connivance" of the Social Communication (journalism program) of the "Universidad Minuto de Dios", Zipaquirá. This context is characterized as largely rural feature that caught my attention as a researcher to investigate what was the proper way to design the course, how students can take the concepts there worked and also if these were concretized into real action after they learned them in class.

In addition to the methodological analysis, this thesis led to a conceptual analysis of the proposed citizenship skills and a critical approach to whether the standards proposed by the Ministerio de Educación Nacional are or not a good input for citizenship education in this context. 
As a result of this investigation it was possible to identify the main curricular path course based on the characteristics of the context and realities of student. Besides these realities possible to understand how appropriate the citizenship skills developed in the classroom and how these are translated into real action in the particular environments of students.

\section{Keywords}

Citizenship, competences, curriculum, higher education, learning.

\section{Um exercício de restruturação curricular no ensino superior para aportar na formação cidadã na Colômbia: um caso no município de Zipaquirá.}

\section{Resumo}

Este trabalho se baseia na reestruturação curricular do curso de "Comunicação, cidadania e convivencia" do programa de Comunicação Social (jornalismo) da Universidad Minuto de Dios, Zipaquirá. Esse contexto se caracteriza por ser em grande medida rural, característica que captou minha atenção como pesquisador para indagar sobre qual era a maneira adequada de desenhar o curso, como os estudantes assumem os conceitos ali trabalhados e se esses são concretizados em ações reais depois de serem aprendidos em a aula.

Além da análise metodológica, esta tese levou a uma análise conceitual das competências cidadãs propostas na aula e uma abordagem crítica sobre o padrões propostos pelo Ministério da Educação Nacional são ou não um bom insumo para a educação cidadã nesse contexto.

Como resultado dessa investigação foi conseguido identificar a principal rota curricular do curso baseado nas características do contexto e realidade dos estudantes. Além estas realidades permitiram entender como se apropriam das 
competências cidadãs desenvolvidas na aula e como estas se convertem em ações reais nos entornos particulares dos estudantes da disciplina.

\section{Palavras-Chave}

Cidadania, competências, currículo, ensino superior, aprendizagem

\section{Introducción}

El presente artículo es resultado de una investigación académica y pretendió un estudio curricular del curso Comunicación, ciudadanía y convivencia, del programa de Comunicación Social (periodismo), Universidad Minuto de Dios, Zipaquirá. Buscó indagar cómo los estudiantes de una población, en su mayoría rural, asumieron los contenidos y las competencias que en esta asignatura fueron brindados.

Para los estudiantes que cursaban esta asignatura y la mayoría de la población de este contexto, Zipaquirá por ser un entorno en su mayoría rural, tiene como sello propio el rótulo de municipio alejado de dinámicas propias de una ciudad, y gracias a esto los procesos ciudadanos y el ejercicio de la participación en la toma de decisiones y en temas de la agenda pública del municipio son casi nulos.

Ante esto, se busca un modelo de ciudadanía que ayude a los estudiantes a comprender a Zipaquirá como una ciudad, partiendo de las competencias ciudadanas propuestas por el Ministerio de Educación Nacional (las competencias ciudadanas son: el conjunto de conocimientos y de habilidades cognitivas, emocionales y comunicativas que, articulados entre sí, hacen posible que el ciudadano actúe de manera constructiva en la sociedad democrática (2004, p. 8) y un trabajo en aula que construyan un esfuerzo por empoderar a la población frente al ejercicio ciudadano.

Como docente de la Universidad realicé un análisis curricular y rediseñé un curso de mi autoría, con el objetivo de crear situaciones de aula apropiadas para la formación ciudadana efectiva. Fueron varias las razones que motivaron este estudio, entre ellas, el mejoramiento del ejercicio académico que ha madurado desde mis estudios profesionales y de posgrado. También el entender cómo los estudiantes que vieron este curso, apropiaron los contenidos del mismo y cómo ejercieron las competencias ciudadanas que fueron socializadas en clase. Este interés surge 
a que durante mi trabajo como docente, identifiqué necesidades como la falta de regulación de actos que atentan contra el buen desarrollo de la ciudadanía en Zipaquirá. Además de esto, es también pertinente para mí como investigador, evaluar curricularmente el curso para determinar la ruta adecuada a seguir en términos de estrategias pedagógicas y procedimentales, de cara al reto de formar profesionales comprometidos con la construcción de una ciudadanía fuerte y sólida.

\section{Marco teórico}

Para hablar de formación ciudadana y de cómo los estudiantes de este contexto entienden su rol como ciudadanos, fue necesario primero establecer un fundamento teórico que permitiera entender el mapa sobre el cual este trabajo se sustentó.

Uno de los conceptos que respaldó el estudio fue el de armonizar ley, moral y cultura propuesto por Antanas Mockus (1999), que se refiere a entender estos tres componentes como sistemas de regulación de la vida en sociedad. La ley, es definida como un sistema jurídico, que se encuentra enmarcado en la constitución política y tiene ciertos alcances, que el pueblo en algún momento de la historia concordó, y que a su vez prevé ciertos castigos y multas ante la violación de los principios inscritos allí, que van en contra de lo que la gente legitima como una conducta dentro de los parámetros establecidos por la ley.

El segundo es la moral, que difiere del sistema jurídico, en cuanto es un proceso interno de reflexión del ser humano que viene, en parte, heredado de y enseñado por los padres, abuelos y generaciones pasadas. De esta manera, su sistema de penalización, también diferente, está más ligado a una serie de sentimientos como: vergüenza, pena, culpa, entre otros. Así, se convierte en un sistema de regulación interno cuando el ser humano utiliza su escala de valores para diferenciar lo bueno de lo malo y en esta coyuntura realiza su plan de vida.

Además de los acuerdos establecidos por el Estado y los que el ser humano construye de manera endógena, existe un sistema de convenios colectivamente establecidos por la comunidad que se convierten en otro nivel de regulación. Este incorpora comportamientos, actitudes, hábitos, entre otros, que funcionan como medición para que el sistema regulador de estos acuerdos, la sociedad, pueda enmarcar las conductas de la gente dentro de lo que culturalmente está aceptado o no, haciendo claro que entre culturas esta normatividad puede cambiar ya que es parte de la identidad propia de una comunidad. 
Después de realizar las definiciones de cada uno de los conceptos, Mockus presenta la idea del divorcio que existe entre estas tres formas de regulación que una sociedad democrática tiene, aumentando así, con esta hipótesis, la necesidad de que la educación para la ciudadanía en este contexto se refuerce, debido a que:

Justamente la falta de congruencia entre la regulación cultural y las regulaciones morales y jurídicas del comportamiento se expresa en Colombia como auge de la violencia, de la delincuencia y de la corrupción; como ilegitimidad de las instituciones, como debilitamiento del poder de muchas tradiciones culturales y como crisis o debilidad de la moral individual (Mockus 1999, p. 21).

Esta falta de congruencia, que se manifiesta como la ausencia de un estado armónico de relaciones interpersonales en cualquier contexto y que consiste en una violación de las formas de regulación, ya sea desde lo cultural (reglas a nivel comunitario), desde lo legal (sistema jurídico que rige todo el país), o desde lo moral (sistema de reglamentación personal que implica un proceso reflexivo e interno), conlleva al deterioro del tejido social y del concepto articulado de ciudadanía, yendo en contravía del desarrollo del contexto, en todos los niveles. Si bien estos niveles: ley, moral y cultura, son diferentes, tienen una relación directa con el reconocimiento propio de un sujeto, es decir, su afiliación consciente y responsable a un espacio o territorio determinado; así pues, el divorcio entre estas, se convierte en una hipótesis para este trabajo investigativo y una evidencia más de que el conflicto social o estructural que vive Zipaquirá puede ser "causado o agravado por limitaciones de la comunicación" (Mockus, 1999, p. 9), ya que las personas no pueden conocer ni dar a conocer el sistema legal bajo el cual se rigen; establecer canales propicios de interrelación ni formas adecuadas de corregir de una manera fraterna actitudes y comportamientos de los demás que puedan perjudicar la sana convivencia ideal en el entorno; $y$, por último, expresar sus ideales y deseos personales para generar una concepción plural de ser ciudadano.

Ante esta necesidad, la comunicación se presenta como un derrotero de la educación ciudadanía debido a que este campo de conocimiento "ayuda a descentrarse lo necesario para comprender cómo mis derechos se relacionan con los de los demás y cómo su respeto se articula al cumplimiento de los deberes de los demás y viceversa" (Mockus, 1999, p. 9). De esta forma el individuo adquiere la capacidad de dejar de pensar en sí mismo, descentrarse, para pasar a entender la realidad desde la multiplicidad de visiones de las cuales puede estar rodeado. 
Nada de esto puede ser logrado si no se piensa en un sistema para relacionarse con los demás, basado en procesos comunicacionales con un fundamento lingüístico, gestual, cognitivo, emocional y de códigos que determinan su buen desarrollo.

De esta manera se hace explícita la relación de la hipótesis realizada por Mockus y expuesta anteriormente con los propósitos de este estudio, que buscó establecer objetivos de aprendizaje en los estudiantes, encaminados a descentrarse a través de la comunicación, para lograr comprender la ley y sus sistemas de regulación; la cultura y los acuerdos que en ella se realizan, que le ayudarán al ciudadano a regular su comportamiento socialmente.

Al lograr una armonización profunda de estas formas de estructurar la vida cotidiana se logra un ambiente democrático ideal para cualquier sociedad. Se asume que la democracia implica cierto tipo de angustia que se genera al tener que decidir por sí mismo entre dos puntos de vista u opciones igualmente válidos, sumando esto a la concepción de actuar bien o mal en determinada situación de la realidad. Como lo planteaba Zuleta (2001):

Pensar por sí mismo es más angustioso que creer ciegamente en alguien. Nombrar algún líder, algún guía cualquiera que sea el nombre que le demos (Hitler, Mao Tse-Tung, Khomeini, etc.), genera un entusiasmo enorme porque libera de la angustia, de la responsabilidad, de la duda sobre si lo que estoy haciendo realmente está bien hecho o no. La palabra del líder nos economiza todos los problemas (p. 99).

El estado ideal del ser humano de lograr esa postura, no se obtiene en solitario, implica inevitablemente la relación constante con el otro, en este sentido:

... La democracia es modestia, disposición a cambiar, disposición a la reflexión autocrítica, disposición a oír al otro seriamente (....). A la angustia y a la modestia, se les suma el exigir respeto, pero se debe aclarar que el respeto sufre reduccionismos que llevan a que este sea malinterpretado y de la misma manera mal exigido. Generalmente, el exigir respeto se ha relacionado con el hecho de que a toda costa sea escuchado y valorado como verdadero y válido el punto de vista propio. Este concepto universalmente manejado genera, por ejemplo, la proliferación de "micro-verdades" que terminan por incrementar la fragmentación de egocentrismos que van en contra de un respeto mutuo legítimo y sensato. El verdadero respeto exige que nuestro punto de vista, sea equivocado total o parcialmente, sea 
puesto en relación con el punto de vista del otro, a través de la discusión (Ibídem, pp. 100-101).

Así pues, la democracia pasando por todas estas cualidades humanas o lo que podríamos traducir de la teoría de Estanislao Zuleta como competencias ciudadanas, se convierte en un proceso evolutivo político y social constituido sobre el plano cultural y mediado por la comunicación. Si este objetivo está atado al que propuso Bobbio (1996), según el cual en la democracia ideal el pueblo era sujeto del poder, mas no objeto del mismo, la ejecución de este modelo puede verse afectado por la evolución de las sociedades, por ejemplo, hacia una sociedad industrializada que deja de centrar su atención en la comunicación interpersonal y pasa a ser un modelo de información masificado que necesita vigilancia y tecnificación. Así pues, el protagonista deja de ser el ciudadano del común y pasa a ser el experto o técnico quien tiene el poder de crear contenidos y decidir sobre la transmisión de los mismos en sus manos.

Con el ánimo de rescatar estos análisis sobre las relaciones más basadas en el ciudadano y menos en la institucionalidad, Andrés Mejía y Betsy Perafán (2006) proponen tres principios que sirven de sustento para la reflexión curricular en el campo de la ciudadanía. Estos son:

1. Las competencias ciudadanas deben estar menos basadas en la adopción por parte de los estudiantes de normas generales éticas o ciudadanas, y más basadas en el desarrollo por parte de ellos de maneras de analizar situaciones y de actuar consecuentemente, de decidir siempre de manera inteligente y sensible.

2. Los estudiantes deben desarrollar un conocimiento profundo acerca de las características particulares de las comunidades y grupos sociales a los que pertenecen, desde las que definen los niveles micro hasta las que definen los niveles macro.

3. Es necesario incluir dentro de la educación ciudadana una educación política que abarque no solo la normatividad para la práctica de la política, sino la práctica misma (Mejia y Perafán, 2006, pp. 32-33). 
Estos principios son una forma de aterrizar a la práctica la teoría de los dos referentes teóricos que se plantearon anteriormente: la consolidación entre ley, moral y cultura; y el concepto de democracia planteado por Zuleta.

Algo importante para tener en cuenta es si se desarrollan en el estudiante de igual forma las competencias ciudadanas en un nivel micro (relaciones interpersonales) o en un nivel macro (relaciones entre grupos sociales), concibiendo que son diferentes y que su manejo y entendimiento conllevan procesos diferentes entre estos dos niveles.

En los contextos micro se encuentran, por ejemplo, la escucha activa, la empatía y el manejo de la ira, grupo propio trabajado en las relaciones interpersonales. Diferentes a las competencias manejadas en un nivel macro tales como la comprensión de los derechos de una comunidad indígena o la ejecución efectiva de sus derechos y deberes civiles a través de unas herramientas determinadas y brindadas por el Estado.

De esta manera, la revisión curricular del curso incluyó un balance justo entre competencias en estos dos niveles: micro y macro, para no caer en el reduccionismo de pensar que "si un ciudadano es competente para entender y actuar apropiadamente con respecto a las relaciones entre personas, individualmente, a nivel micro, entonces será también competente para entender y actuar apropiadamente con respecto a las relaciones entre grupos sociales" (Ibídem p. 28). Esta es una situación que la educación para la ciudadanía debe atender para que los comportamientos de las personas en ambos contextos permitan las relaciones armónicas y así una ciudadanía óptima.

De manera especial se tuvo en cuenta el programa de estándares en competencias ciudadanas del MEN, debido a que este es una apuesta a nivel nacional sobre educación en ciudadanía y era necesario que este estudio estuviera alineado a los esfuerzos del Gobierno Nacional de entender el proceso de enseñanza aprendizaje en estos términos.

El programa del Ministerio consistió en proponer estándares básicos de formación en ciudadanía en la educación básica y media, que parte de considerar este tipo de enseñanza "como un proceso que se puede diseñar, con base en principios claros, implementar, con persistencia y rigor, evaluar continuamente e involucrar en los planes de mejoramiento de cada institución" (Ministerio de Educación Nacional, 2004, p. 5). De esta forma, el trabajo diseñado por el MEN fue un insumo para llevar estas investigaciones realizadas de la educación básica y media a la educación superior y formación profesional en el municipio, particularmente en 
el curso que se analizó, rediseñando objetivos y estrategias para la formación en ciudadanía.

El plan de acción propuesto por el Ministerio de Educación Nacional, que es novedoso en el municipio de Zipaquirá debido a que no ha sido aplicado ningún estudio como este, plantea que es necesario, al igual que al aprender matemáticas, seguir un proceso que puede ser planeado, operado y posteriormente evaluado, basado en competencias ciudadanas comunicativas, cognitivas y emocionales.

Las competencias comunicativas son definidas como aquellas que son necesarias para sostener una comunicación adecuada, tanto de manera interpersonal como grupal, y se entienden como "la capacidad [del individuo] para escuchar atentamente los argumentos ajenos y para comprenderlos, a pesar de no compartirlos. O la capacidad para poder expresar asertivamente, es decir, con claridad, firmeza y sin agresión, los propios puntos de vista" (Ibídem, 2004, p. 13).

Lo cognitivo está referido al proceso de aprehensión de conceptos y procesamiento de información que una persona pueda hacer durante su vida y específicamente a lo siguiente:

La capacidad para realizar diversos procesos mentales, fundamentales en el ejercicio ciudadano. Por ejemplo, la habilidad para identificar las distintas consecuencias que podría tener una decisión, la capacidad para ver la misma situación desde el punto de vista de las personas involucradas, y las capacidades de reflexión y análisis crítico, entre otras (Ibídem, p. 12).

Las necesidades en este entorno pasan más allá de ser solo comunicativas y cognitivas, por eso, veo imprescindible el desarrollo de otro tipo de competencias como las emocionales, las cuales se definen como:

Las habilidades necesarias para la identificación y respuesta constructiva ante las emociones propias y las de los demás. Por ejemplo, la capacidad para reconocer los propios sentimientos y tener empatía, es decir, sentir lo que otros sienten, por ejemplo su dolor o su rabia (Ibídem, 2004, p. 13).

Para este tipo de sociedad es que un currículo de educación en ciudadanía debe estar dirigido, para enfrentar una humanidad fragmentada, para que tome en sus manos los sistemas de regulación (legales, morales y culturales) ayudada por la formación de las competencias ciudadanas que propone el MEN buscando ser una manera de enfrentar la vida cotidiana en la actualidad en el municipio de Zipaquirá. 


\section{Metodología}

\section{Diseño}

El propósito de esta investigación fue realizar una revisión curricular del curso "Comunicación, ciudadanía y convivencia", para precisar qué herramientas pedagógicas eran apropiadas para desarrollar competencias ciudadanas. A partir de esta innovación se evaluó cómo los estudiantes se empoderaron de los contenidos de clase y, de la misma forma, cómo tradujeron dicho discurso desde lo académico a sus relaciones diarias y a su cotidianidad.

Esta evaluación partió de la revisión del currículo trabajado hasta ese momento en la asignatura ubicada en quinto semestre para determinar si la ruta pedagógica era o no la adecuada para las necesidades de los estudiantes. Esto implicaba el desarrollo y la aplicación de nuevas herramientas metodológicas y de acciones evaluativas que permitieran dar cuenta de si el proceso estaba dando o no resultado. La metodología usada fue cualitativa, debido a que se llevó a cabo en un contexto natural, en el cual los actores, docente y estudiantes, utilizaron la Investigación Acción "como un medio para orientar la planeación de la acción social organizada, que se encauza a la transformación de algún tipo de realidad social le que resulta insatisfactoria al grupo humano involucrado en el proceso investigativo" (Casilimas, 1996).

\section{Contexto y participantes}

Los participantes de esta investigación fueron 28 estudiantes pertenecientes a esta asignatura, jóvenes entre los 20 y 24 años de edad, de estratos socio-económicos 1 y 2.

Una de las limitaciones que pudo tener el estudio es que debido a la figura de docente de la asignatura que poseía, además de la de investigador, existió la posibilidad de algunas respuestas sesgadas a algunas de las preguntas realizadas en las sesiones de trabajo, por el temor de ser víctimas de represalias, situación que por supuesto, viciaría de alguna manera, los resultados. Ante esta condición, los participantes fueron invitados por medio de un formato de consentimiento que garantizó confidencialidad y voluntariedad en el proceso. Además, la relación de cercanía y confianza entre investigador y sujeto de investigación, propia de los 
estudios cualitativos, fue fundamental desde el inicio para que las características propias tanto de ellos como las del contexto no se modificaran durante el trabajo y los resultados fueran más significativos.

\section{Rediseño curricular}

La restructuración curricular buscó analizar, en principio, componentes teóricos de la asignatura para encontrar si la ruta actual propuesta por el programa era la adecuada, tenía los contenidos que el estudiante y su contexto inmediato necesitaban y si las competencias actitudinales, procedimentales y cognitivas eran las apropiadas para trabajar. Otro frente de reflexión de este trabajo fue la renovación metodológica de la planeación por clases, para que la metodología diera al estudiante la posibilidad del conocimiento de las competencias ciudadanas y a su vez de la acción sobre las mismas en la realidad.

Esta evaluación, apoyada con los métodos de recolección de información, permitieron contestar las preguntas de investigación atendiendo: necesidades curriculares, las formas de recepción de los contenidos de la clase y cómo se llevan estos a la aplicación real, a través del diálogo, la problematización de la realidad, entre otros conocimientos teóricos y prácticos que un buen ejercicio ciudadano requiere.

\section{Métodos de recolección de información}

Debido al carácter cualitativo de la investigación, fue perentorio hacer uso de varias herramientas de recolección de información para responder las preguntas planteadas inicialmente. Entre estas se utilizaron grupos focales, debido a que durante el recorrido del estudio se debía contar con parte del grupo para conocer sus opiniones compartidas sobre la estructura del curso y, sobretodo, sobre el desarrollo de las competencias que se perseguían, atendiendo a "su carácter colectivo, que contrasta con la singularidad personal de la entrevista en profundidad" (Ibídem, p. 145).

Particularmente esta herramienta ayudó a responder la primera pregunta de investigación planteada, debido a que este método permitió la reflexión colectiva acerca del glosario de temas tratados en la asignatura, haciendo que todos los actores del contexto se empoderaran del proceso de reflexión curricular y además 
poner en común necesidades e intereses que ayudaran a la reconstrucción de la asignatura.

De la misma forma se utilizaron entrevistas individuales que permitieron ahondar en algunos casos particulares en los que fue necesario hacerlo, debido a que las condiciones de la persona (barrio o vereda, edad, estrato, afiliación política, entre otras) ayudaron a responder de una forma más puntual algunas indagaciones del estudio. Específicamente, se buscó conocer cómo los estudiantes ejecutaron o hicieron realidad las competencias ciudadanas que en clase se discutieron y formaron, una de las preguntas de investigación que este estudio se propuso resolver.

Asimismo y yendo de la mano con las preguntas de investigación, las reflexiones del investigador sobre el proceso que fueron un aporte significativo para el estudio pudieron servir como evaluación del mismo, fueron sistematizadas por medio de diarios de campo, debido a que son una fuente importante de información para la evaluación de las metodologías y contenidos propuestos para el curso. Este ejercicio no fue posible sin antes hacer una revisión documental del currículo que está compuesto básicamente por: la estructura de contenidos que recoge la esencia de la asignatura (síntesis, justificación, objetivos, competencias a desarrollar, etc.) y la estructura de créditos, tabla que discrimina las sesiones de clase durante el semestre, el contenido de la clase y la competencia que se desarrolla en la misma.

\section{Resultados y conclusiones}

El proceso de investigación que me llevó a una significativa serie de aprendizajes, dio inicio a su fase de aplicación de la innovación pedagógica y de recolección de información en enero de 2013, permitiéndome dar respuesta a las preguntas de investigación que me planteé inicialmente y que me permito citar:

1. ¿Cuál es la ruta curricular adecuada para la enseñanza de competencias ciudadanas en un contexto como Zipaquirá?

2. ¿Cómo los estudiantes del curso "Comunicación, ciudadanía y convivencia" asumen y se apropian de las concepciones ciudadanas que son brindadas en clase? 
3. ¿Cómo el ejercicio ciudadano es llevado del aula a los contextos inmediatos de cada uno de los estudiantes?

Estas preguntas surgieron basadas en una hipótesis que tuve como investigador del divorcio existente entre los tres sistemas de regulación planteados por Antanas Mockus (1999) y que en Zipaquirá son un eventual detonante para el ejercicio ciudadano inapropiado.

Se propuso como solución la aplicación de un programa de clases que incluía innovaciones pedagógicas y metodológicas, apoyado en el argumento de entender la formación ciudadana "como un proceso que se puede diseñar, con base en principios claros, implementar, con persistencia y rigor, evaluar continuamente e involucrar en los planes de mejoramiento de cada institución" (MEN, 2004, p. 5).

En cuanto a la formación por competencias no solo busca la adquisición de saberes, sino que persigue de la misma manera un saber hacer que el modelo praxeológico promulgado por la universidad refuerce de la siguiente manera:

La responsabilidad social, expresada en una sensibilidad consciente y crítica frente a la problemática de las comunidades y del país y en unas competencias para el trabajo de promoción del desarrollo humano y social, se construye a través de un proceso praxeológico, investigativo y de realización personal que se realiza desde las prácticas sociales y profesionales, e integra los conocimientos adquiridos en la academia con las experiencias de interacción y compromiso social suscitadas en la práctica y el contacto con las comunidades (Vicerrectoría General Académica, 2004).

Metodológicamente el curso fue rediseñado pensando en superar la dirección que tenía de ir de lo teórico a lo práctico, pasando así a una estructura mixta en la que se trabajaran teoría y práctica a la vez para poder fortalecer ese vínculo entre la conceptualización de las competencias y su aplicación en situaciones reales que hicieran que el aprendizaje fuera significativo para los estudiantes.

Esta situación se evidenció en el paso de la segunda a la tercera clase, en cuanto la primera se tornó muy conceptual cuando a los estudiantes les fue pedido que leyeran material bibliográfico y definieran de manera individual preguntando a personas con las que convivieran usualmente el concepto de ciudadanía. Finalmente lo que sucedió en cuanto a la conceptualización de los estudiantes fue que se redujeron a resumir la postura del autor sin hacer un análisis profundo de lo que les fue pedido. 
Esto resultó siendo algo esquemático para ellos y su participación fue alta en la medida en que tal como lo evidencio en uno de los diarios de campo que diligencié: compartían sus conceptos con el resto de la clase, mientras yo como docente intervenía para hacer algunas aclaraciones sobre lo mencionado, los estudiantes al parecer privilegiaron los conceptos que arrojé y que el autor hacía en su artículo, por encima de los que ellos encontraron dentro de sus investigaciones, lo que convirtió una clase que estaba pensada como participativa, en una clase magistral e inclusive unidireccional.

Observando esto y tomando como referencia el primer principio que consideran cruciales Mejía y Perafán para acercarse a la formación en competencias ciudadanas, al que hice referencia en el marco teórico, emprendí la renovación metodológica que permitiera que el aprendizaje de los estudiantes estuviera centrado en su propia voz y en su propia perspectiva más allá de lo que dijera yo como profesor.

A partir de lo anterior, diseñé para la tercera sesión una actividad basada en la lectura de dos textos, se dividía la clase en dos grupos y estos debían redactar 5 preguntas con el objetivo de que fueran ellos los actores del proceso de aprendizaje y además que la discusión los ayudara a generar un aprendizaje colaborativo. En la segunda parte de la dinámica, cada grupo debía construir y redactar un escrito basado en la siguiente pregunta: ¿cómo la comunicación ayuda a construir la ciudadanía?

La actividad permitiría que los conceptos que fueran adquiridos en la clase anterior entraran en la escena del aprendizaje y así poder ser aprehendidos por los estudiantes, con esto además el principio de aprendizaje significativo tomaría fuerza cuando se privilegiaran el poder que tenían los estudiantes de determinar qué conceptos y aprendizajes adquirían del material otorgado y sobretodo que participaran activamente tanto en los grupos formados como en la parte de las preguntas y respuestas. Esto llevó a resultados como los que se consignan en uno de los diarios de campo y que a continuación cito: la dinámica de la clase cambió drásticamente con el cambio de metodología, la participación aumentó exponencialmente frente a lo que habían sido las dos primeras clases de corte magistral. Inclusive este método permitió que algunos de los estudiantes que anteriormente no participaron, en este caso lo hicieran haciendo unos aportes de gran relevancia.

En las respuestas a la entrevista realizada, los estudiantes reconocieron que el currículo cambió y que esto permitió que ellos como actores principales del proceso de enseñanza aprendizaje, llevaran una ruta clara hacia los objetivos propuestos 
por la clase apoyados en el plan curricular. A continuación presento algunos ejemplos de lo que se manifestó en relación a esto.

Por los códigos de ética de mantener la confidencialidad de los nombres de los estudiantes me referiré a ellos como estudiante A, B, C, etc. El estudiante A entrevistado, privilegió el salón de clase como un espacio idóneo para la promoción de la discusión sobre temas que en otros contextos diferentes a este no podrían hacerlo, sea porque no tienen la oportunidad o porque no era de su interés: "esta estructura ha hecho que nosotros como estudiantes tengamos más participación en un espacio no necesariamente que sea afuera, sino pues nosotros colectivamente como estudiantes podemos opinar sobre cualquier cosa que está ocurriendo" (entrevista al estudiante A).

La interacción que se daba a la hora de crear conocimiento colectivo en el salón de clase, como se evidencia en el fragmento anterior, y el componente práctico de las actividades le daban una mayor significancia y reflexión a lo aprendido como lo muestra otra de las entrevistas realizadas: "es muy interesante que cada actividad esté planteada de una forma más dinámica y más de interacción que de otra cosa, lo que permite que estemos actuando sobre lo que aprendemos permanentemente" (entrevista al estudiante B).

El ejemplo de participación colectiva en la vereda (Río Frío - Zipaquirá) mencionado por un estudiante en una de las clases y que a continuación citaré de uno de los diarios de campo, es una prueba de que sí existe una preocupación por temas públicos pero que las formas de comunicación y conocimiento entre lo local (una vereda como esta) y lo global (la ciudad, las instituciones educativas, el mismo gobierno municipal y regional) no funcionan muy bien o no existe ninguna forma de hacerlo, y estas experiencias se pierden, o más bien, se quedan en los contextos inmediatos creando unos prejuicios frente a los actores públicos en este tipo de comunidades.

En el caso del lugar de mi casa pues es un lugar que hace parte de una vereda pero hay otra que la está reclamando (en Río Frío, Zipaquirá), entonces hay otra vereda que se llama San Jorge a la cual se supone pertenece ese lugar, sin embargo Rio Frío está reclamando este sector, como finalmente no se llegó a un acuerdo, no se pudo durante más de 15 años llegar a un acuerdo, las personas del sector decidieron unirse todas y convocaron una asamblea para decir -nosotros lo que vamos a tener es como cierta autonomía, nos independizamos- pasaron la propuesta de lo que se podía hacer, el por qué ellos lo hacían, se enumeraron 
las razones y realmente se llegó a un acuerdo e incluso se llegaron a hacer cosas que hace quince años no se podían. Las personas de Río Frío con las de San Jorge hasta se mataban por considerarse de un barrio o del otro y se logró llegar a mediar, entonces por eso digo que la participación ciudadana está mal vista, se cree que porque son campesinos o tenemos la idea que el que es ciudadano es el que está en la ciudad y no, realmente no, eso es un concepto errado" (fragmento de una de las clases).

Estas definiciones que los estudiantes hacen en una clase participativa en la que el debate depende de ellos, hace que analicen su realidad, sus construcciones simbólicas, sus relaciones, sus cotidianidades a partir de cómo los medios comunicativos actúan y crean unos referentes allí, lo que permite resaltar el segundo principio que es necesario para acercarse a una formación ideal de la ciudadanía y que menciona que "los estudiantes deben desarrollar un conocimiento profundo acerca de las características particulares de las comunidades y grupos sociales a los que pertenecen, desde las que definen los niveles micro hasta las que definen los niveles macro" (Mejía y Perafán, 2006).

Como otro componente de la innovación pedagógica propuesta, estuvo la tarea de hacer un diario de campo sobre la visita a un lugar de la ciudad que ellos escogieran y que fuera analizado bajo las formas de ciudadanía que ellos identificaran. De esta manera, los estudiantes podrían reconocer cómo los procesos ciudadanos eran vividos en la cotidianidad, entre otros, de la plaza de mercado, la Catedral de Zipaquirá, el Terminal de Transporte.

Este ejercicio fue crucial en la innovación, en la medida en que los estudiantes lograron identificar y relacionar sitios que usualmente transitan con unos procesos de construcción ciudadana que va más allá de lo político y comienzan a ligarlo con la serie de relaciones que allí se dan, que van desde las comerciales hasta las espirituales, pasando por las comunicacionales, entre otras. Este análisis que fue consignado en cada uno de los escritos, también permitió ahondar en las características micro que tenía en particular este grupo social y a las que hacen referencia Mejía y Perafán a la hora de estudiar un contexto y sus procesos ciudadanos.

De esta manera, y con la firme convicción de intervenir intencionalmente en el diseño de las actividades del curso para lograr uno de los objetivos del estudio, se realizó una actividad evaluativa (primer parcial) de tres puntos en los que se combinaba lo teórico y lo práctico, construyendo en grupo los conceptos de liderazgo, ciudadanía y convivencia, y poniéndolo en práctica en ese mismo momento. 
Lo que permitió esta forma de evaluación fue que se privilegiaran el componente humano y comunicativo del ejercer ciudadano, dándole una nueva mirada al tema que logró involucrar a los estudiantes alrededor de un diálogo de saberes y formación colectiva de competencias ciudadanas, demostrando que "la comunicación, a través de este tipo de dinámicas de construcción simbólica contribuye a reconstruir y a crear nuevos referentes de certeza y de acción colectiva" (Bayuelo, Cadavid, Durán, González, Tamayo y Vega, 2008).

Al terminar esta sesión de manera informal, algunos estudiantes se acercaron a manifestar el agrado que habían sentido con respecto a la evaluación y de la misma manera cómo estas dinámicas les habían permitido comprender y empoderarse de las competencias que se perseguían en ese primer corte.

Este punto del ejercicio investigativo fue importante para abrir la caja de herramientas de recolección de información y hacer entrevistas, debido no solo a que era el final del primer corte académico sino que además consideré que las acciones realizadas y las innovaciones hechas podrían brindar algunos resultados relevantes para el análisis y que ayudarían a responder las preguntas de investigación.

Una de estas preguntas apuntaba al hecho de poder generar que los estudiantes hicieran una conexión lógica y significativa entre la teoría y la práctica. Por eso tomé la decisión de incidir en el currículo y cambiar el formato del primer parcial, lo que llevó a los estudiantes a tener apreciaciones como la siguiente:

Las clases y las temáticas que se han venido presentando y que están incluidas dentro de este plan curricular, han tenido primero que todo una consecución que es importante para que nosotros como estudiantes podamos llevar cierta línea conductora sobre lo que estamos aprendiendo (entrevista al estudiante B).

Además de tener clara la línea o el camino de aprendizaje que se había pactado desde el principio, los estudiantes lograron interesarse en los temas de la clase y sobre todo en hablar de ellos, tal como lo menciona el siguiente estudiante en su entrevista:

Antes y al momento de iniciar este curso uno se preguntaba, ipara qué una clase de ciudadanía? Sin embargo lo que ha planteado el profesor ha sido muy útil, utilizar estas herramientas y audiovisuales ha ayudado a que uno se interese en participar en estas clases, entonces yo si pienso tal vez que como empezamos a cómo vamos ahora ha servido mucho saber lo que es ciudadanía, porque 
personalmente iba a una clase de ciudadanía pero era igual hablar y hablar el profesor y uno qué hace en esa clase. Pero ya digamos con las nuevas herramientas que se han desarrollado han servido para que uno como estudiante le interese participar (entrevista al estudiante A).

Para los estudiantes, las clases donde se generaban debates fueron un aliciente para desarrollar algunas competencias ciudadanas como la argumentación, la toma de decisiones, la escucha activa, entre otras; además de este ejercicio de apropiación, reconocen que si son trabajadas de la manera adecuada, estas serán replicables fuera del salón de clase.

Una de las actividades que más tuvo repercusión en este tipo de habilidades y que generó más participación en el salón de clase fue el juego de roles propuesto por Parra (2004). Esto sirvió como ejemplo para hablar de la herramienta metodológica que pone en situación a los estudiantes con respecto a un conflicto entre los dueños de negocios y los residentes de un barrio que se niegan a que estos abran sus negocios en este sitio. La relevancia de esta actividad a nivel educacional es que se basa en los siguientes principios pedagógicos tales como el aprendizaje a través de la acción y la interacción social.

Otro de los estudiantes quien participó en el estudio se refirió al juego de roles que se realizó durante una de las clases del segundo corte académico de la siguiente manera:

El promover la participación incluye también tener en cuenta por ejemplo el voto, cuando se realizó la actividad del juego de roles de los dueños de los bares y los representantes de los residentes, donde se dio primero una problemática, cada persona o cada grupo tomaba una decisión referente a la situación, entonces era como promover esa participación del ciudadano dando cuenta que acá nos desarrollamos como estudiantes y a su vez somos ciudadanos, era como ponernos en un papel que de una u otra forma se vive a diario (testimonio del estudiante $D$ en el grupo focal).

La toma de decisiones, en algunos casos a nivel grupal, se convirtió este semestre en una competencia determinante para el desarrollo de las clases en concordancia con la competencia argumentativa y el pensamiento crítico para poder hacer un buen ejercicio ciudadano, como lo manifiestan en los diferentes momentos en los que tuve un contacto con ellos (entrevistas, grupos focales). 
Esta actividad fue escogida con el objetivo de responder al último de los principios que Mejía y Perafán mencionan como fundamental para acercar a los estudiantes a los conceptos claves de la ciudadanía', recordando que "es necesario incluir dentro de la educación ciudadana una educación política que abarque no solo la normatividad para la práctica de la política, sino la práctica misma" (Mejía y Perafán, 2006).

Al parecer actividades como esta sí fomentan en los estudiantes una actitud ciudadana con competencias, como la toma de decisiones, la argumentación, la asertividad, la escucha activa entre otras, que les permiten resolver situaciones reales.

El siguiente apartado de la entrevista con el estudiante A, es una evidencia clara de que la intervención funcionó en términos curriculares y que sí se generaron espacios de participación en los que los estudiantes, con situaciones cercanas a su realidad, podían poner en práctica las competencias que en ese momento se querían formar.

Nos metimos mucho en el rol como ciudadanos afectados y otros que defendían los intereses por parte de los negocios, entonces esto ayudó a que nosotros entendiéramos también un poco lo que de verdad estaba ocurriendo y apropiarnos de las cosas porque de lo que ocurre alrededor uno tiene que estar haciéndose partícipe para dar solución a este problema y la herramienta que utilizamos nosotros fue el diálogo a través de una junta, digámoslo así, por medio de un moderador obviamente expusimos nuestras partes para al final llegar a un acuerdo sin utilizar hechos violentos sino utilizando el dialogo como una herramienta de solución a un problema (entrevista al estudiante A).

Todas estas acciones que pertenecen a la reestructuración curricular realizadas durante estas 16 sesiones de clase, eran el propósito principal de esta investigación, los análisis de las mismas y las evidencias que fueron obtenidas con las herramientas de recolección de información y que fueron presentadas anteriormente permitieron evaluar de una manera positiva la reestructuración planteada en cuanto a que los resultados obtenidos con el curso se pudieron verificar y los objetivos del mismo se reconocen como alcanzados por los estudiantes.

Otra forma de poder medir el éxito de la reestructuración, tal como se había planteado desde la formulación del proyecto, es el efecto que tuvieron las actividades en los sistemas de regulación de los estudiantes, sobre todo en el moral y en el 
hecho de propiciar cambios en la interpretación que un individuo hace del sistema cultural, lo que puede convertirse en la motivación que ellos tengan en un futuro para tener una incidencia en la regulación legal que rige este contexto.

En primera medida, las actividades además de reforzar algunas de las competencias ciudadanas, funcionaban como un llamado de atención frente a algunas actitudes negativas de los estudiantes tanto en el salón de clase como en su cotidianidad, entre otras, la falta de escucha activa y la disposición a las diferentes posturas que podrían tener sus compañeros.

Estos cuestionamientos sobre algunas actitudes negativas dentro del aula surgieron cuando en una entrevista un estudiante mencionó:

Lo que más se evidencia y el temor que tenemos es la burla, no tal vez el comentario, sino la burla que dijo esto pero no tenía nada que ver con lo que estábamos hablando, tal vez es eso que no genera esa confianza a la hora de participar (entrevista al estudiante A).

Luego, y sin alguna intencionalidad de por medio, el estudiante B en un momento de su entrevista menciona:

Esas actividades (haciendo referencia a las que se llevaron a cabo este semestre) fueron bastante claras y lo llamaron a uno y le dijeron "oiga hermano, usted es un ciudadano y debe responder de esta forma", la actuación correcta de un ciudadano es esta, entonces creo que fue clara tanto en lo que se quería como en lo que se vivió (entrevista al estudiante B).

De esta manera, se evidencia cómo los sistemas de regulación, en particular el sistema moral, entraban en juego y realmente eran un factor determinante a modificar con la reestructuración de la ruta curricular y con las innovaciones metodológicas y pedagógicas.

La idea de crear una conciencia y llamar la atención de los demás a hacerlo puede ser una forma en la que el individuo cambie su interpretación del sistema cultural, que si bien surge desde el sistema de regulación moral a través de un proceso reflexivo, puede actuar en consonancia con esa nueva postura formada para intervenir en un nivel ya no endógeno sino exógeno. En el grupo focal uno de sus participantes mencionó: 
Yo decía: "para qué votar", mal acto, yo no puedo decir eso porque si me sigo quejando de que las vías están en mal estado o de cualquier cosa, pues el cambio depende de mí, y si nosotros tenemos ese espacio y no lo aprovechamos, ¿cómo queremos vivir bien? Eso ya depende mucho más de nosotros, de generar esa conciencia de participación, nosotros hacemos parte de una ciudadanía, si participamos o no estamos afectando directa o indirectamente, si quiero que este país progrese, no solo yo sino todos, es solo cuestión de participar (estudiante F, grupo focal).

Más allá de generar y motivar una conciencia colectiva en el municipio, algunos de los estudiantes buscan con los aprendizajes del curso y el ejemplo de otros compañeros la participación en espacios que les permitan de alguna manera materializar el esfuerzo por un buen ejercicio ciudadano, tal como otro estudiante lo mencionó en el grupo focal:

Yo creo que como ciudadano uno puede aportar como lo hizo mi compañera frente a un compromiso con los otros ciudadanos, con los que nosotros nos enfrentamos a diario y con los que viven en nuestro entorno, sería una buena opción uno participar en una JAC donde uno pueda tener el criterio de participación y frente a una problemática que haya, uno pensar qué solución puede dar frente a ella y cooperar y tener un compromiso no solo con uno mismo sino con las personas con que uno convive a diario (estudiante $\mathrm{G}$, grupo focal).

Las actitudes y comportamientos de los estudiantes que se podrían entender también como los sistemas de regulación de cada uno de ellos, demuestran que ante la hipótesis del divorcio entre ley, moral y cultura existente en el municipio de Zipaquirá, el esfuerzo de plantear proyectos como esta investigación para incidir en el sistema de regulación moral de los estudiantes de educación superior y eventualmente en presentarles a ellos alternativas sobre cómo actuar para regular a otros desde lo cultural, son válidos en cuanto apunten a una formación ciudadana significativa, que en este caso llevó consigo un análisis y aterrizaje adecuado al contexto de los estándares propuestos por el Ministerio de Educación, apoyado en la idea de que formar en ciudadanía implica algo más que un aprendizaje político y meramente centrado en las normatividades establecidas por la ley, y que por medio de una serie de desarrollos pedagógicos innovadores que se conviertan en significativos para los estudiantes se logre la consolidación de una educación para 
la ciudadanía madura y fundamentada en principios pedagógicos que privilegien la acción humana y las competencias comunicativas, cognitivas y emocionales que le permitan al ser humano un ejercicio ciudadano, si bien no utópico, ideal para enfrentar los retos que la sociedad actual implican.

\section{Referencias Bibliográficas}

Bayuelo, S., Cadavid, A., Durán, O., González, A., Tamayo, C. A., y Vega, Y. (2008). Lo que le vamos quitando a la guerra. Bogotá: Friedrich Ebert Stiftung.

Bobbio, N. (1996). El futuro de la democracia. México: Fondo de Cultura económica.

Casilimas, C. A. (1996). Investigación cualitativa. Bogotá: ARFO.

Chaux, E., Lleras, J., y Velásquez, A. M. (2004). Competencias ciudadanas: de los estándares al aula, una propuesta de integración a las áreas académicas. Bogotá: Uniandes, MEN.

Mejía, A., y Perafán, B. (2006). Para acercarse al dragón, para amansarlo, es necesario haberlo amansado primero: una mirada crítica a las competencias ciudadanas. Revista de Estudios Sociales, 23-35.

Ministerio de Educación Nacional. (2004). Formar para la ciudadanía sí es posible, lo que necesitamos saber y saber hacer. Bogotá: MEN.

Mockus, A. (1999). Armonizar ley, moral y cultura.

Vicerrectoría General Académica. (2004, abril). Modelo educativo universitario. Bogotá: Sistema Universitario Uniminuto.

Zuleta, E. (2001). Educación y democracia un campo de combate. Medellín: Hombre Nuevo. 